\title{
SELENIUM IN CERTAIN IGNEOUS ROCKS
}

\author{
TAPIO Koljonen
}

Koljonen, Tapio 1973: Selenium in certain igneous rocks. Bull. Geol. Soc. Finland 45, 9-22.

The selenium contents of the igneous rocks from Finland and Iceland and a few from West-Greenland studied in this paper can be tentatively summed as follows:

The selenium content shows a general tendency to decrease when passing from a basic to a more siliceous rock.

Excluding ultrabasic rocks, this statement can also be expressed by saying that the selenium content exhibits a positive correlation with the colour index of the rock. Selenium very probably does not enter the structures of the common rock-forming minerals but is largely contained in the accessory sulphide phase. The distribution of selenium between the various calc-alkalic igneous rocks mainly reflects the abundance of the sulphide phase in the rocks.

The volcanic rocks of Iceland are generally more rich in selenium than their abyssal equivalents in the Finnish Precambrian. This fact could possibly result from the slowly advancing crystallisation of the igneous rock masses under deep-seated conditions, thus offering a better opportunity for thsulphide-bearing emanations to escape from the magma and enter the surrounding bedrock.

Adopting the standard section constituting the upper continental earth's crust, tentatively estimated by Wedepohl $(1969,244)$, the data of Table 1 yields a value of $47 \mathrm{ppb}$ as the arerage selenium content of abyssal igneous rocks. Using the average of two parts acidic (granite) to one part basic (gabbro) rock, the average selenium content of abyssal igneous rocks amounts to 58 ppb. Both these average estimations are near the value $50 \mathrm{ppb}$ presented by Turekian and Wedopohl (1961) and by Vinogradov (1962).

Tapio Koljonen, Department of Geology and Mineralogy, University of Helsinki, 00170 Helsinki 17, Finland.

\section{Introduction}

The mode of occurrence of selenium in Nature is largely dominated by the close relationship between selenium and sulphur. In sulphide de- posits of relatively low selenium concentration this element enters the structures of the sulphides where it substitutes for sulphur. In some cases the selenium content of the sulphides may range up to a few percent; scarcely any sulphide 
minerals are known in which selenium is absent. If the $\mathrm{Se} / \mathrm{S}$ ratio of the environment exceeds a certain limit, then selenium occurs as specific selenides which may or may not form a solid solution with the sulphides of analogous composition. The mineralogy of selenium and the occurrence of the element in sulphides is covered by considerable literature, the most comprehensive reviews of which have been published by Sindeeva (1964) and Tischendorf (1966).

Excluding the occurrence of selenium in sulphide deposits, the analytical data for the general geochemistry of the element are restricted mainly to soils, waters and various sedimentary materials. In this connexion, the reader is referred only to the bibliography compiled by Luttrell (1959).

The information dealing with the abundance of selenium in igneous rocks is scanty (Goldschmidt and Hefter 1933; Goldschmidt and Strock 1935; Goldschmidt 1958, 532-540). Davidson and Powers (1959) reported a considerable amount of analytical data for the volcanics of the western United States and Hawaii. The contents found are mostly given as less than $1 \mathrm{ppm} \mathrm{Se}$ and are generally lower for the crystalline volcanic rocks than for the ashes connected with them. Sindeeva (1964) published a series of 18 selenium determinations made on various igneous rocks of the USSR indicating a range of variation from 0.1 to $0.37 \mathrm{ppm} \mathrm{Se}$, the figures given by her are, on the average, slightly high. The reason for this circumstance is probably the fact that she worked mainly with volcanic and dyke rocks where the selenium content is higher than in their plutonic equivalents. Sindeeva also had the disadvantage that she was compelled to work near the lower limit of detection of her analytical method.

Brunfelt and Steinnes (1967) gave the selenium contents of eight standard rock samples, their analyses being made using the highly sensitive neutron activation method. For comparison with the data of this paper their values are reproduced here:

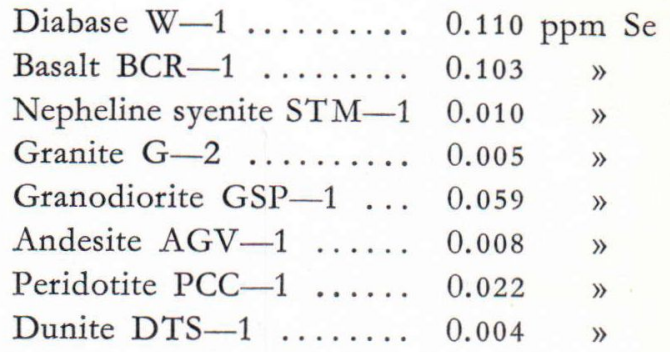

In addition, some data for selenium in igneous rocks is also scattered in the literature. The references can be found in the papers mentioned.

The average abundance of selenium in igneous rocks has been estimated by the following authors:

Vogt (1898) ............ n $\cdot 10^{-7 \%} \mathrm{Se}$

Clarke and Washington (1924) $\mathrm{n} \cdot 10^{-6}$

Vernadsky (1924) ........ 10 10-4 $-10^{-5}$

Behrend and Berg (1927) ... 2.5 $10^{-6}$

Noddack and Noddack (1936) $8 \cdot 10^{-5}$

Goldschmidt (1935) ...... 9. $9 \cdot 10^{-6}$

Vinogradov (1949) $\ldots \ldots \ldots .6 \cdot 10^{-5}$

Vinogradov (1956) $\ldots \ldots \ldots . \mathrm{n} \cdot 10^{-6}$

Turekian and Wedepohl (1961) $\quad 5 \cdot 10^{-6}$

Vinogradov $^{1}(1962) \ldots \ldots \ldots \quad 5 \cdot 10^{-6}$

\footnotetext{
1 Igneous rock mixture containing two parts of acid to one part of basic rock.
}

Some of these values have been adopted in a number of later reviews and compilations. The scarcity of the original analytical data underlying these figures makes these selenium abundance estimations nothing more than "good guesses». For this reason a study of selenium in igneous rocks was undertaken by the author mainly using specimens from Finland and Iceland and a few from West-Greenland. The results of the study are presented in the following.

\section{Sampling and analytical procedure}

Most of the specimens of Finnish rock used in this work were collected on field trips in various parts of the country. The author was privileged to be assisted, during the collection, 
by colleagues who had mapped the areas concerned and who generously assisted in selecting exposures at which the rocks were considered typical. Consequently, many of the specimens are derived from spots described in the literature. Some other areas, however, were visited without local guidance. A part of the specimens used had been placed at the author's disposal from the collections of the Geological Survey of Finland and the Department of Geology of the University of Helsinki. The specimens from Iceland were collected on a guided field trip in 1968 and those from West Greenland during the author's field work in 1966, carried out jointly with Mr. S. Väisänen, of the Technical University of Helsinki.

A list of the specimens of igneous rocks used and the results of the selenium determinations are compiled in Table 1 . When applicable, references to the localities and to the petrological characteristics of the specimens have been added to the table.

The analytical procedure used for the quantitative selenium determination will be described in detail later, and consequently only a brief summary of the method is reported.

From each specimen, ca. $300 \mathrm{~g}$ was ground in a vibrating mill (»Schwingmühle» manufactured by Messrs. Siebtechnik G.M.B.H., Mülheim, West Germany). This type of mill is reported as producing a minimum contamination. To test the effect of possible contamination from the mill and from the reagents used, blank analyses were made at intervals during the work and many of the analyses were repeated. The blank analyses were partly made by adding known amounts of selenium to the charge. The reproducibility was found to be within $\pm 10 \%$ (Koljonen 1965).

Briefly, the analytical procedure was as follows: A sample of $20-70 \mathrm{~g}$, on average $50 \mathrm{~g}$, was decomposed by fusing with $\mathrm{NaOH}-\mathrm{Na}_{2} \mathrm{O}_{2}$ in a nickel crucible. To prevent evaporation of selenium, $5 \mathrm{~g} \mathrm{Fe}_{2} \mathrm{O}_{3}$ was added to the charge. The cake was dissolved in sulphuric acid and the selenium was distilled quantitatively from the solution to which $\mathrm{HBr}-\mathrm{Br}_{2}$ had been added. Arsenious acid was added to the distillate and the selenium was collected by co-precipitation with metallic arsenic in reducing conditions. Selenium was finally determined colorimetrically as diphenylpiazselenol.

The selenium contents given in Table 1 are expressed in $\mathrm{ppb}\left(1 \mathrm{ppb}=0.001 \mathrm{ppm}=10^{-7} \%=\right.$ $1 \mathrm{mg} /$ ton). The concentration given in the table corresponds to $1 \mu \mathrm{g}$ in the charge which, for an average charge of $50 \mathrm{~g}$, corresponds to $20 \mathrm{ppb}$ in the rock. This value amounts to about twice the background obtained in the blank determinations, viz., $0.5 \mu \mathrm{g}$. Selenium concentrations lower than $20 \mathrm{ppb}$ are marked »n.d.» (not detected) in the table.

\section{Plutonic rocks}

On the basis of the data summarised in Table 1 , the selenium contents of the rocks belonging to the calc-alkalic igneous rock suite are diagrammatically presented in the histogram of Fig. 1. The following specimens have been included in this figure:

Ultrabasic rocks .......... Nos. 1- 11

Gabbroic rocks ......... 12- 32

Dioritic rocks ......... 33-39

and $59-72$

Granitic rocks (excluding rapa-

kivi granites) $\ldots \ldots \ldots \ldots \ldots . \quad 73-109$

Finnish rapakivi granites .... 110 128

It is understood that, especially in the intermediate to acidic rock groups mentioned, some of the rocks studied may not be strictly magmatic in origin. A few remarks may be added concerning these groups. The gabbroic rock group also includes anothosites, the selenium contents in the anorthosites studied (Nos. 2732) tend to be lower than those of the different gabbro varieties (Nos. 12-26). The difference in the spread of the selenium contents between the anorthosites and the gabbros proper is, however, not sufficiently pronounced to warrant 
presentation of the anothosites as a separate rock group. In a qualitative manner, both in the anorthosites and in the gabbros proper, the selenium content shows a positive correlation with the colour index of the rock.

In general, the darker the rock, the higher its selenium content. The dioritic group ranges from quartz-free or slightly quartz-bearing varieties to granodiorites. As in the gabbroic rocks, the selenium content of the dioritic rocks shows a tendency to increase with the colour index. Among the granitic rocks the rapakivi granites were distinguished as a separate group.

Inspection of the histogram in Fig. 1 reveals the fact that the selenium content found varies within relatively wide limits in most of the rock groups concerned. In addition, the ranges of variation overlap considerably and thus, on the basis of the data availabale, a presentation of the average selenium contents of the calc-alkalic igneous rock groups in the conventional manner is considered only of limited value. With this reservation in mind, the following averages are tentatively given:

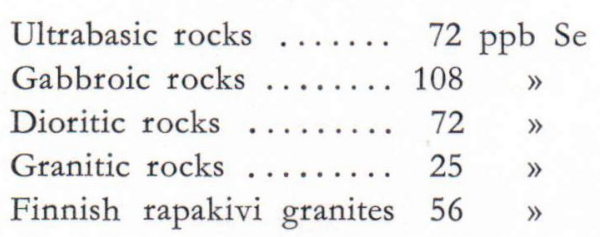

In their order of magnitude these figures compare reasonably well with those listed by Brunfelt and Steinnes (1967) for the standrad igneous rock samples, reproduced in the introduction of this paper. The figure for the ultrabasic rocks is, however, clearly higher than those given by these authors for peridotite and dunite, respectively.

Despite the merely semi-quantitative character of the average selenium contents listed above, the histogram of Fig. 1 makes it evident that selenium becomes somewhat enriched in the ultrabasic to intermediate rocks, preferably in the polymineralic gabbros. The main dark, iron rich
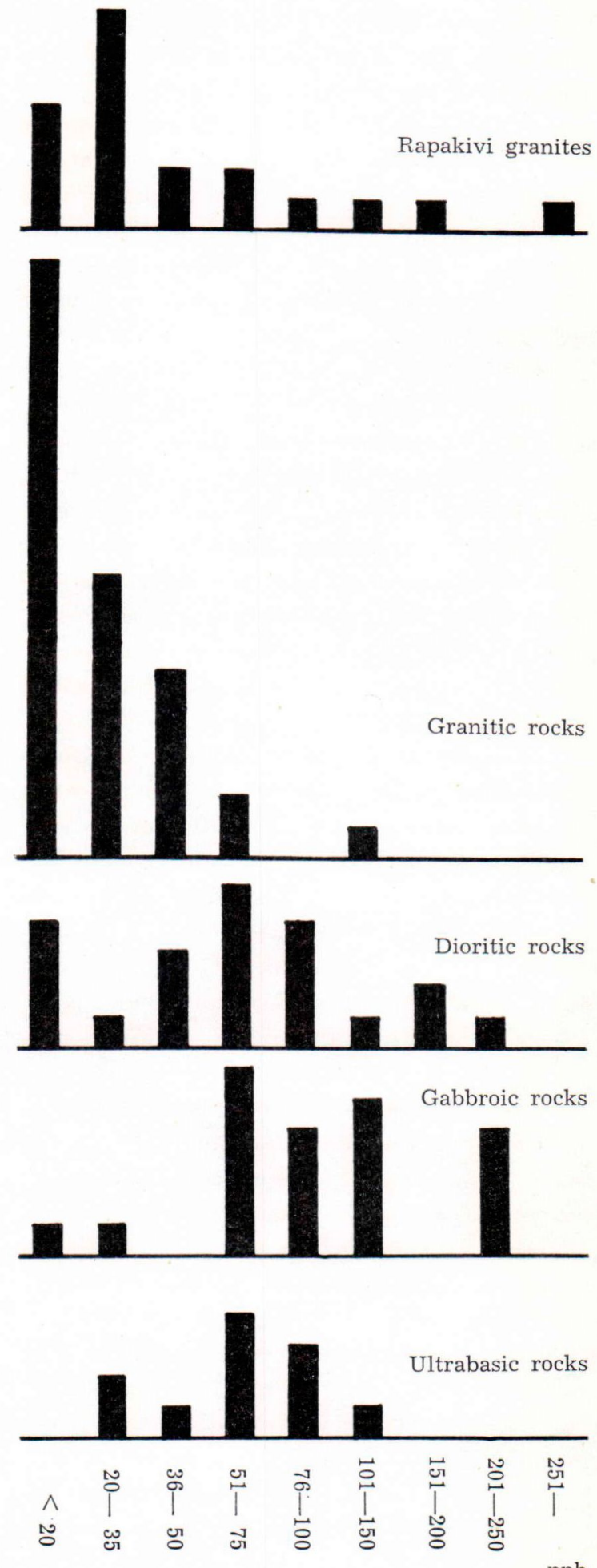

$\mathrm{ppb}$

Fig. 1. Review of the selenium contents in the plutonic rocks studied ( one sample) 
constituents of such rocks are represented by amphiboles (hornblende) and/or micas (biotitephlogopite) which are both known to be capable of incorporating a diversity of trace elements in the structure. No studies dealing with the partition of selenium between the different melanocratic rock-forming silicate minerals are, however, known to the author. The ultrabasics of the Sukkertoppen complex in West Greenland (Nos. 4-10) show a fairly narrow range of variation in their selenium content despite the considerable mineralogical diversity exhibited by these rocks. In this connexion it is worth noting that the silicate-phosphate rocks of the Siilinjärvi carbonatite complex, which contain phlogopite, amphibole or serpentine as the sole silicate constituents, (Nos. 50-54) are decidedly more rich in selenium than the carbonate-apatite rocks of the same complex (Nos. 55-58).

$A$ very pronounced feature in the distribution of selenium among the rock groups of Fig. 1 is its improverishment in the precambrian granitic rocks of Finland(excluding the rapakivi granites). In more than half of the specimens analysed the selenium content is below the limit of detection.

The data explained above seems to indicate that the behaviour of selenium in magmatic differentiation is not governed by any incorporation into the structures of the main silicate constituents. Fig. 1 can be more plausibly explained by assuming that the enrichment or depletion of selenium in a particular rock has little or nothing to do with the main silicate phases. The paramount feature of the geochemistry of selenium in the evolution of calcalkalic magmas is apparently its incorporation in the sulphide phase in which it substitutes for sulphur. The rocks in which the sulphide phases are more abundant, as in many gabbroic rocks, are enriched in selenium. The Finnish precambrian granitic rocks are usually devoid of sulphides.

In contrast to the other granitic rocks of the Finnish precambrian studied, the selenium content of the specimens of rapakivi granite analysed varies within a more extended range. The population of the lowest concentration ranges in Fig. 1 is less pronounced for the rapakivi granites than for the other granitic rocks. This circumstance is possibly due to the fact that the rapakivi granites represent intrusions posterior to the metamorphism of the surrounding bedrock and thus have better retained their selenium content.

\section{Hypabyssal and dyke rocks}

The selenium contents of the hypabyssal and dyke rocks analysed are summarised in the histogram of Fig. 2. The following specimens have been included in this figure:

Various dyke rocks ...... Nos. 129-145

Pegmatites and aplitic veins $\quad 146-167$

Acid porphyries ....... 168-175

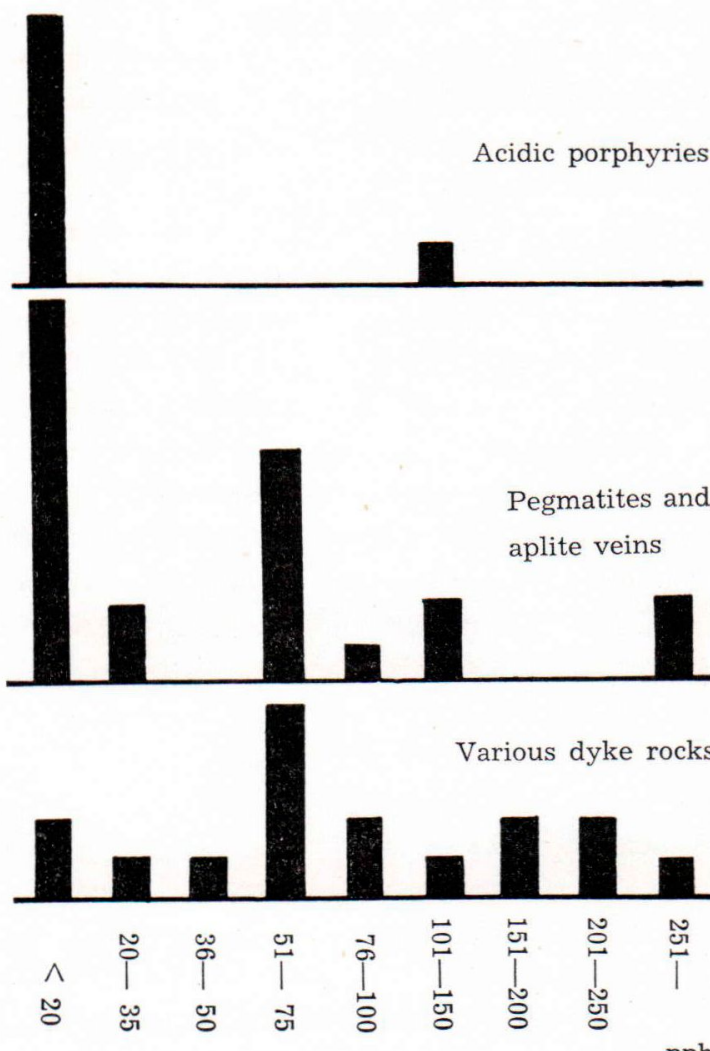

$\mathrm{ppb}$

Fig. 2. Review of the selenium contents in the hypabyssal and dyke rocks studied ( 
The specimens of the acidic porphyries analysed belong to the suite of the rapakivi granites and represent porphyritic dykes cutting the rapakivi itself. It is interesting to note that these porphyries are strongly depleted in selenium (Fig. 2), even more so than the "granitic rocks» of Fig. 1. This depletion is remarkable because the rapakivi granites, the host of the porphyries, show a tendency to contain more selenium than the other granitic rocks of the Finnish Precambrian. The porphyry dykes apparently offered more or less open channels along which selenium was able to escape. The only high-selenium porphyry in Fig. 2 (No. 168 in Table 1) represents the glassy margin of a dyke the core of which (No. 169) is devoid of selenium. The margin probably was chilled and consolidated sufficiently rapidly to retain the selenium.

On the other hand, the pegmatites and the aplitic veins studied display a frequency pattern (Fig. 2) which resembles that of the rapakivi granites. The data seems to confirm the known tendency of selenium to become enriched in pegmatitic and hydrothermal crystallizations of a granitic magma.

The various dyke rocks comprise lamprophyres, diabases, plagioclase porphyries etc. mainly from the Finnish Precambrian. Judging from the field relations none of these dyke rocks (excluding No. 144) are genetically connected with the rapakivi granites. The dyke rocks are often remarkably enriched in selenium, especially the lamprophyres and the olivine-free diabases. The range of variation of the selenium content is, however, quite variable and the histogram of Fig. 2 exhibits a rather evenly distributed frequency pattern.

\section{Volcanic rocks}

The volcanic rocks studied originate from Iceland and consist of lavas and pyroclastics. Two main groups are distinguished and the selenium contents presented in the histograms of Fig. 3:
Acidic volcanics

from Iceland

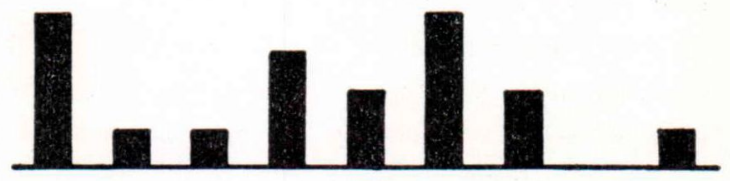

Basaltic and andesitic volcanics

from Iceland

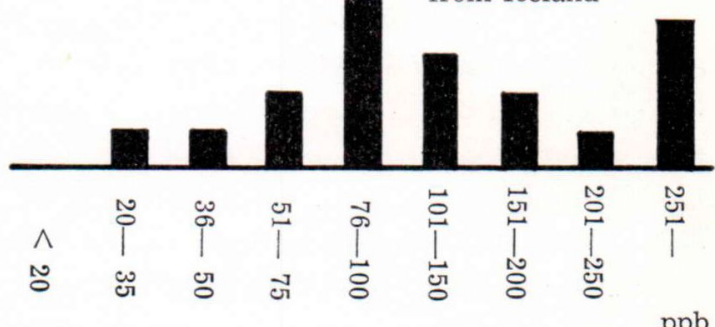

$\mathrm{ppb}$

Fig. 3. Review of the selenium contents in the volcanic rocks studied (one sample)

Basaltic-andesitic

volcanics ....... Nos. 176-195; 211-212

Acidic volcanics .... 197-210; 213-216

The difference between the frequency patterns of these two groups is considerably less pronounced than that between those of the deepseated granitic and gabbroic rocks of the Finnish precambrian. The rhyolites (Nos. 197-200) show relatively high values for selenium, higher than most of the obsidians analysed (Nos. 202-207), and especially higher than the rhyolitic ashes (Nos. 213-216). The selenium contents of the basalts (and andesites) show wide variation with only a few examples, however, in the lowest concentration range. In contrast to the rhyolitic ashes, the two specimens of basaltic ash (Nos. 211-212) show very high values for selenium.

It seems possible that the selenium content in these volcanics is, to a considerable extent, contained in the vesicles of the rock. This possibility is indicated by specimen No. 182 (130 $\mathrm{ppb} \mathrm{Se}$ ) which is highly vesicular compared with specimen No. 188 (78 ppb Se) which represents a compact and dense variety of the same lava. 
Another example is represented by specimen No. 202 (280 ppb Se), a highly vesicular core of an obsidian vein, compared with specimen No. 207 (selenium not detected) which represents a dense and glassy margin of the same vein.

The enrichment of selenium in the volcanic exhalations is demonstrated by specimen No. 177 (360 ppb Se), a basaltic lava collected in the immediate vicinity of an active vent and burned red by the escaping gases. Specimen No. 218 (3 $700 \mathrm{ppb} \mathrm{Se}$ ) of sulphur from a hot spring and specimens Nos. 219-220 (860 and 260 ppb Se, respectively) represent further examples illustrating the enrichment of selenium in the exhalative crystallizations.

Acknowledgements - Professor Kalervo Rankama, University of Helsinki, directed the interests of the author to the present study.

The laboratory work was carried out in the laboratory of the Department of Geology and Mineralogy of the University of Helsinki.
I take great pleasure in thanking Professors Martti Saksela, Mauno Härme and Heikki V. Tuominen, Directors of the aforesaid Department, for their great liberality and kindness in allowing me freely to use their Department.

I am deeply indepted to Professor Th. G. Sahama for his keen interest in the present investigation, for much advice during the various stages of the work, for many a fruitful conference, and for criticism of the manuscript.

I wish to express my gratitude to numerous colleagues, especially to Mr. Ossi Näykki, Lic. Phil., University of Helsinki, who have helped to carry out the present investigation.

Mr. Olavi Y. Nurminen assisted me in handling the samples and Mr. Urpo Eklund drew the histograms.

Mr. John Nelson, Lic. Tech., checked the language of the English manuscript.

Financial support for this study was provided by grants awarded by the Finnish Cultural Foundation (Suomen Kulttuurirahasto)and the Foundation for the Investigation of Natural Resources in Finland.

Finally, I would like record my warm appreciation to my wife, Ritva, for her patience and support during the various laborious phases of my work. 
Table 1.

Selenium contents of the igneous rocks

\begin{tabular}{|c|c|c|c|c|}
\hline No. & Rock and locality & $\begin{array}{l}\text { Selenium } \\
\text { content } \\
\text { ppb }\end{array}$ & No. & Rock and locality \\
\hline & Ultrabasic rocks & & 38 & Quartz monzodiorite. Keuruu, Lihjamo, Finland \\
\hline 1 & $\begin{array}{l}\text { Peridotite, porphyritic. Orimattila, Mallusjoki, Finland } \\
\text { (Laitakari and Simonen 1962; Laitakari and Simonen } \\
\text { 1963, 19 Anal. 2) }\end{array}$ & 104 & 39 & $\begin{array}{l}\text { (Marmo 1963 a; } 1963 \mathrm{~b}, 30-32 \text {, Table IX, Anal. 3) } \\
\text { Diorite. Siilinjarrvi, Sarkkilampi, Finland (Puustinen } \\
\text { 1968, 15-17; 1969, 90; and 1971) }\end{array}$ \\
\hline 2 & Peridotite. Mäntsälä, Soukkio, Finland (Kaitaro 1956) & 70 & & Monzonite \\
\hline 3 & $\begin{array}{l}\text { Peridotite. Pinlajavesi, Finland (Marmo 1963 b, 26, } \\
\text { Anal. 1) } \ldots \ldots \ldots \ldots \ldots \ldots \ldots \ldots \ldots \ldots \ldots \ldots \ldots \ldots \ldots \ldots \ldots\end{array}$ & 34 & 40 & Monzonite. Brändö, Ava, Finland (Kaitaro 1953, \\
\hline 4 & $\begin{array}{l}\text { Olivinite, hyperstehene-and chromite-bearing. Sukker- } \\
\text { toppen, Itipilua, Greenland (Väisänen 1967, 47-50) }\end{array}$ & 75 & & 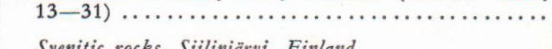 \\
\hline 5 & $\begin{array}{l}\text { Olivinite, tremolite- and phlogopite-bearing. Sukker- } \\
\text { toppen, Itipilua, Greenland (Väisänen } 1967,39-47 \text { ) }\end{array}$ & 72 & 41 & $\begin{array}{l}\text { Syenitic rocks, Sitlinjärvi, Finland } \\
\text { Syenite. Pahkalampi (Puustinen 1968, 62-68; 1969; }\end{array}$ \\
\hline 6 & $\begin{array}{l}\text { Olivinite, chromite-bearing. Sukkertoppen, Itipilua, } \\
\text { Greenland (Väisanen } 1967,39-47) \ldots \ldots \ldots \ldots \ldots\end{array}$ & 40 & 42 & 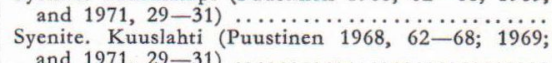 \\
\hline 7 & $\begin{array}{l}\text { Dunite. Sukkertoppen, Itipilua, Greenland (Väisänen } \\
1967,26-38 \text { ) } \ldots \ldots \ldots \ldots \ldots \ldots \ldots \ldots \ldots \ldots \ldots \ldots \ldots \ldots \ldots \ldots \ldots \ldots \ldots \ldots \ldots \ldots \ldots \\
\text { Dunite, metamorphosed to tremolite-antigorite rock. }\end{array}$ & 95 & 43 & $\begin{array}{l}\text { and 1971, 29-31) } \\
\text { Syenite (unakite). Kuuslahti (Puustinen 1968, 62-68; } \\
1969 \text {; and 1971, 29-31) }\end{array}$ \\
\hline${ }^{\circ}$ & 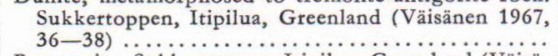 & 98 & 44 & $\begin{array}{l}\text { Syenite. Siilinjärvi railway cut (Puustinen } 1968,62-68 \text {; } \\
\text { 1969; and } 1971,29-31) \ldots \ldots \ldots \ldots \ldots \ldots \ldots \ldots\end{array}$ \\
\hline 9 & $\begin{array}{l}\text { Pyroxenite, Sukkertoppen, Itipilua, Greenland (Väisä- } \\
\text { nen } 1967,11-14) \ldots \ldots \ldots \ldots \ldots \ldots \ldots \ldots \ldots \ldots \ldots\end{array}$ & 75 & 45 & $\begin{array}{l}\text { Syenite. Asikkala (Puustinen 1968, 62-68; 1969; and } \\
1971,29-31) \ldots \ldots \ldots \ldots \ldots \ldots \ldots \ldots \ldots \ldots \ldots \ldots \ldots \ldots \ldots\end{array}$ \\
\hline 10 & $\begin{array}{l}\text { Pyroxenite, anortite-bearing. Suk kertoppen, Itipilua, } \\
\text { Greenland (Väisänen 1967, } 11-14 \text { ) } \ldots \ldots \ldots \ldots \ldots\end{array}$ & 90 & 46 & $\begin{array}{l}\text { Melasyenite. Pahkalampi (Puustinen 1968, 74-75; } \\
\text { 1969; and 1971, 29-31, 34, Anal. 1) } \ldots \ldots \ldots \ldots \ldots\end{array}$ \\
\hline 11 & $\begin{array}{l}\text { Hornblendite. Pihtipudas, Mäntymäki, Finland (Ny- } \\
\text { känen } 1963,21,35 \text {, Anal. 1) } \ldots \ldots \ldots \ldots \ldots \ldots \ldots \ldots \ldots\end{array}$ & 34 & & Alkali rocks of Iivaara, Finland \\
\hline & Gabbros & & $\begin{array}{l}47 \\
48\end{array}$ & $\begin{array}{l}\text { Ijolite. Kuusamo (Lehijärvi } 1960,32-35) \ldots \ldots \ldots \ldots \\
\text { Ijolite. Kuusamo (Lehijärvi } 1960,32-35 \text { ) }\end{array}$ \\
\hline 12 & $\begin{array}{l}\text { Gabbro. Hyvinkää, Finland (Hackman 1905, 55; } \\
\quad \text { Kaitaro 1956) } \ldots \ldots \ldots \ldots \ldots \ldots \ldots \ldots \ldots \ldots \ldots \ldots \ldots \ldots \ldots \ldots \ldots \ldots \ldots \ldots\end{array}$ & 230 & 49 & Melteigite. Kuusamo (Lehijarvi $1960,35-38$ ) ....... \\
\hline 13 & $\begin{array}{l}\text { Gabbro. Hyvinkää, Finland (Hackman 1905, 55; } \\
\quad \text { Kaitaro 1956) } \ldots \ldots \ldots \ldots \ldots \ldots \ldots \ldots \ldots \ldots \ldots \ldots \ldots \ldots \ldots \ldots \ldots \ldots \ldots \ldots \ldots\end{array}$ & 215 & 50 & $\begin{array}{l}\text { Carbnnatite complex of Siilinjärvi, Finland } \\
\text { Serpentine rock. Pahkalampi (Puustinen 1969; and }\end{array}$ \\
\hline 14 & $\begin{array}{l}\text { Gabbro. Kemiö, Rosendal, Finland (Seitsaari } 1955 \text {; } \\
\text { Härme } 1958 \text { b; and } 1960,26) \ldots \ldots \ldots \ldots \ldots \ldots \ldots\end{array}$ & 210 & 51 & $\begin{array}{l}1971,25) \\
\text { Phlogopite rock. Särkilampi (Puustinen } 1969 \text {; and }\end{array}$ \\
\hline 15 & Gabbro. Kuru, Syvälänkylä, Finland (Matisto 1960) & 200 & & 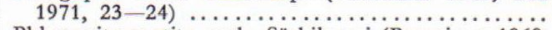 \\
\hline 16 & 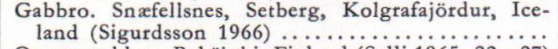 & 135 & 52 & $\begin{array}{l}\text { Phlogopite-apatite rock. Särkilampi (Puustinen 1969; } \\
\text { and } 1971,19,23-24) \ldots \ldots \ldots \ldots \ldots \ldots \ldots \ldots \ldots\end{array}$ \\
\hline $\begin{array}{l}17 \\
18\end{array}$ & $\begin{array}{l}\text { Quartz gabbro. Pyhäjoki, Finland (Salli 1965, 22-27) } \\
\text { Pyroxene gabbro. Virrat, Kotala, Finland (Marmo }\end{array}$ & 104 & 53 & $\begin{array}{r}\text { Amphibole rock. Särkilampi (Puustinen 1969; and } \\
1971,19)\end{array}$ \\
\hline 19 & $\begin{array}{l}1965 \text { a; and } 1965 \mathrm{~b}, 33-35) \\
\text { Gabbro. Hyvinkää, Kytäjä, Finland (Härme 1953, }\end{array}$ & 104 & 54 & $\begin{array}{l}\text { Apatite-phlogopite rock. Särkilampi (Puustinen 1969; } \\
\text { and } 1971,14-19,23-24) \ldots \ldots \ldots \ldots \ldots \ldots \ldots \ldots \ldots\end{array}$ \\
\hline 20 & & 99 & 55 & $\begin{array}{l}\text { Carbonate-apatite rock. Särkilampi (Puustinen 1969; } \\
\text { and 1971) } \ldots \ldots \ldots \ldots \ldots \ldots \ldots \ldots \ldots \ldots \ldots \ldots \ldots \ldots \ldots \ldots\end{array}$ \\
\hline 21 & $\begin{array}{l}\text { Härme } 1958 \text { b; and } 1960,26,61) \ldots \ldots \ldots \ldots \ldots \\
\text { Olivine gabbro. Pihtipudas, Mäntymäki, Finland ( } \mathrm{Ny}-\end{array}$ & 98 & 56 & $\begin{array}{l}\text { Carbonate-apatite rock. Särkilampi (Puustinen 1969; } \\
\text { and 1971) } \ldots \ldots \ldots \ldots \ldots \ldots \ldots \ldots \ldots \ldots \ldots \ldots \ldots \ldots \ldots \ldots\end{array}$ \\
\hline 22 & $\begin{array}{l}\text { känen } 1962 \text {; and } 1963,21,35 \text {, Anal. 2).......... } \\
\text { Hornblende gabbro, near the contact against gneiss. }\end{array}$ & 87 & 57 & $\begin{array}{l}\text { Carbonate rock. Särkilampi (Puustinen } 1969 \text {; and } 1971 \text {, } \\
21-23) \ldots \ldots \ldots \ldots \ldots \ldots \ldots \ldots \ldots \ldots \ldots \ldots \ldots \ldots \ldots\end{array}$ \\
\hline & $\begin{array}{l}\text { Kangaslampi, Rauhamäki, Finland (Kurki 1964, } \\
17-31) \ldots \ldots \ldots \ldots \ldots \ldots \ldots \ldots \ldots \ldots \ldots \ldots \ldots \ldots \ldots \ldots \ldots \ldots \ldots \ldots \ldots\end{array}$ & 82 & 58 & $\begin{array}{l}\text { Carbonate rock. Särkilampi quarry (Puustinen 1969; } \\
\text { and } 1971,21-23) \ldots \ldots \ldots \ldots \ldots \ldots \ldots \ldots \ldots \ldots \ldots \ldots \ldots \ldots \ldots\end{array}$ \\
\hline $\begin{array}{l}23 \\
24\end{array}$ & $\begin{array}{l}\text { Gabbro. Mäntsälä, Soukkio, Finland (Kaitaro 1956) } \\
\text { Gabbro. Mäntsälä, Soukkio, Finland (Kaitaro 1956) }\end{array}$ & $\begin{array}{l}58 \\
58\end{array}$ & & (2) \\
\hline 25 & $\begin{array}{l}\text { Norite. Kinnula, Kivijärvi, Finland (Nykänen 1963, } \\
17-20) \ldots \ldots \ldots \ldots \ldots \ldots \ldots \ldots \ldots \ldots \ldots \ldots \ldots \ldots \ldots \ldots \ldots \ldots\end{array}$ & 52 & 59 & $\begin{array}{l}\text { Quartz diorite. Kalajoki, Finland (Salli } 1955 \text {; and } 1961 \text {, } \\
33-34,45) \ldots \ldots \ldots \ldots \ldots \ldots \ldots \ldots \ldots \ldots \ldots \ldots \ldots \ldots \ldots \ldots \ldots \ldots\end{array}$ \\
\hline 26 & $\begin{array}{l}\text { Gabbro, leucocratic. Snxfellsnes, Lýsuskard, Iceland } \\
\text { (Sigurdsson 1966) } \ldots \ldots \ldots \ldots \ldots \ldots \ldots \ldots \ldots \ldots \ldots \\
\text { Anorthosites }\end{array}$ & n.d. & 60 & $\begin{array}{l}\text { Quartz diorite, porphyritic. Lappeenranta, Lappee, } \\
\text { Saikkola, Finland (Vorma 1964; and } 1965,27-35 \text {, } \\
\text { 67) } \ldots \ldots \ldots \ldots \ldots \ldots \ldots \ldots \ldots \ldots \\
\text { Quartz diorite. Ylöjärvi, Ylinen Finland (Simonen }\end{array}$ \\
\hline 27 & $\begin{array}{l}\text { Anorthosite gabbro. Pihtipudas, Mäntymäki, Finland } \\
\text { (Nykänen } 1963,21,35 \text {, Anal. } 3 \text { ) } \ldots \ldots \ldots \ldots \ldots \ldots\end{array}$ & 140 & 62 & 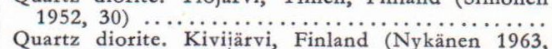 \\
\hline 28 & $\begin{array}{l}\text { Anorthosite. Pertunmaa, Haapasaari, Finland (Savo- } \\
\text { lahti } 1956,19-29 \text {; and } 1966,173-197) \ldots \ldots \ldots\end{array}$ & 130 & 63 & $\begin{array}{l}21-25) \ldots \ldots \ldots \ldots \ldots \ldots \ldots \ldots \ldots \ldots \ldots \ldots \ldots \ldots \ldots \ldots \ldots \ldots \ldots \ldots \ldots \ldots \ldots \\
\text { Quartz diorite. Lavia, Finland }\end{array}$ \\
\hline 29 & $\begin{array}{l}\text { Anothosite gabbro. Karjaa, Mustio, Hállsnäs. Finland } \\
\text { (Härme 1954, 34-38, 43; and } 1960,26) \ldots \ldots \ldots \ldots\end{array}$ & 69 & & Matisto $1952 \mathrm{a}$; and $1952 \mathrm{~b}, 31-38) \ldots \ldots \ldots \ldots$ \\
\hline 30 & Anorthosite. Kemi, Finland (Näykki 1964, 59-65, & & & Granodiorites \\
\hline 31 & $\begin{array}{l}\text { Anorthosite, party saussuritized. Kemi, Finland } \\
\text { (Näykki } 1964,59-65, \text { Anal. 16) } \ldots \ldots \ldots \ldots \ldots \ldots \ldots\end{array}$ & 34 & 64 & $\begin{array}{l}\text { Granodiorite, porphyritic, contains } x \text { xenoliths. Nokia } \\
\quad \text { N-side, Finland (Matisto } 1961 ; 1962,118-120 \\
\text { and } 1968,7) \ldots \ldots \ldots \ldots \ldots \ldots \ldots \ldots\end{array}$ \\
\hline 32 & $\begin{array}{l}\text { Bytownitite. Ylämaa, Tervalainen, Finland (Wahl } \\
\text { 1925, 15-21; Rankama 1944, 32) } \ldots \ldots \ldots \ldots \ldots \ldots \\
\text { Diorites }\end{array}$ & 72 & 66 & 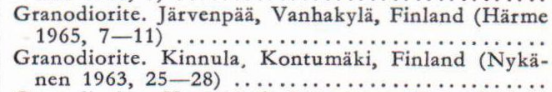 \\
\hline 33 & $\begin{array}{l}\text { Diorite, cummingtonite-bearing. Petäjävesi, Vanha } \\
\text { Puttola, Finland (Rouhunkoski 1959, 61-64) } \ldots\end{array}$ & 228 & 67 & 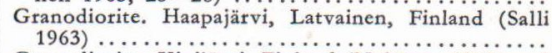 \\
\hline 34 & $\begin{array}{l}\text { Diorite, cummingtonite-bearing. Petäjävesi, Puttola, } \\
\text { Finland (Rouhunkoski } 1959,61-64) \ldots \ldots \ldots \ldots \ldots\end{array}$ & 176 & 68 & $\begin{array}{l}\text { Granodiorite. Kivijärvi, Finland (Nykänen 1962; and } \\
\quad 1963,25-28) \ldots \ldots \ldots \ldots \ldots \ldots \ldots \ldots \ldots \ldots \ldots \ldots \ldots\end{array}$ \\
\hline 35 & $\begin{array}{l}\text { Diorite. Siilinjärvi, Finland (Puustinen } 1968,15-17 \text {; } \\
\text { 1969, 90; and 1971) ...................... }\end{array}$ & 120 & 69 & $\begin{array}{l}\text { Granodiorite. Nurmijärvi, Palojoki, Finland (Härme } \\
1958 \text { a) } \ldots \ldots \ldots \ldots \ldots \ldots \ldots \ldots \ldots \ldots \ldots \ldots \ldots \ldots \ldots \ldots \ldots\end{array}$ \\
\hline 36 & $\begin{array}{l}\text { Diorite. Jyväskylä, quarry near Pohjanlahti, Finland } \\
\text { (Frosterus 1900; and 1903, 102; Hall 1936, 78) }\end{array}$ & 90 & 70 & $\begin{array}{l}\text { Granodiorite. Kerava, Yli-Kerava, Kaskela, Finland } \\
\text { (Härme } 1958 \text { a) } \ldots \ldots \ldots \ldots \ldots \ldots \ldots \ldots \ldots \ldots \ldots \ldots \ldots \ldots \ldots\end{array}$ \\
\hline 37 & $\begin{array}{l}\text { Diorite. Keuruu, Haapamäki, Finland (Marmo } 1963 \text { a; } \\
\text { and } 1963 \mathrm{~b}, 28 \text {, Table VI, 30-32, Anal. 2) ..... }\end{array}$ & 52 & 71 & $\begin{array}{l}\text { Granodiorite. Teisko, Värmälä, Finland (Seitsaari } \\
1951,77-80) \ldots \ldots \ldots \ldots \ldots \ldots \ldots \ldots \ldots \ldots \ldots \ldots \ldots\end{array}$ \\
\hline
\end{tabular}

Selenium $\mathrm{ppb}$ 


\begin{tabular}{c|c|}
\hline No. & Rock and locality \\
\hline 72 & Granodiorite, contains quartz veins, Hyvinkää, Fin-
\end{tabular}
Granodiorite, contains quartz veins. Hyvinkää, Fin-

Granites

73 Orbicular granite. Kuru, Parkusjärvi, Finland (Simo-

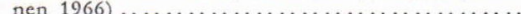

74

75

76

77

78

79

80

81

82

83

84

85

86

87

88
Granite. Suomenniemi, Finland (Frosterus 1900; and 1903, 41-42; Simonen and Tyrväinen 1965) .... Granite. Kuopio, Neulamäki, Finland (Preston 1954,

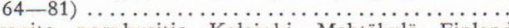
Granite, porphyritic. Kalajoki, Mehtäkylä, Finland

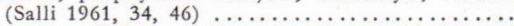
Granite, »Kakola-granite», contains cordierite and garned porphyroblasts. Lemu, Järäinen, Finland (Hietanen 1947, 1073-1078; Härme 1965, 17-19)

Charnockite. Naantali, Ukkopekka bridge, Finland (Hietanen 1947, 1032, 1035-1051)

Granite. Espoo, Bodom, Finland (Erämetsä, Sahama and Kanula 1943, 83; Rankama 1943, 37-38; and 1946; Härme 1960, 36; Marmo and Siivola 1966, 170; Härme 1969)

Granite, porphyritic Jämsänkoski, Koskenpää, Finland (Frosterus 1903, 43-45) ........................... Granite. Jämsänkoski, Koskenpää, Finland (Frosterus

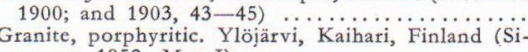
monen 1952, Map I)

Granite, porphyritic. Kalajoki, Mehtäkylä, Finland

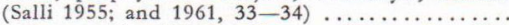
Granite, porphyroblastic. Keuruu, Haapamäki, Finland (Marmo $1963 \mathrm{a}$; and 1963 b, 31, Anal. 6, 34-39) Orbicular granite. Espoo, Nuuksio, Pitkäjärvi, Finland (Simonen 1941, 129-136) ................... Granite. Iisalmi, Paloisvuori, Finland (Wilkman 1931,

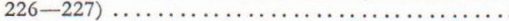
Granite, porphyritic. Petäjävesi, Kintaus, Finiand

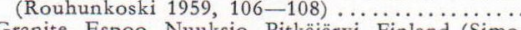
Granite. Espoo, Nuuksio, Pitkäjärvi, Finland (Simo-

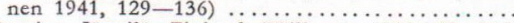
Granite. Jäppilä, Finland (Wilkman 1938, 92, 113) Trondhjemite. Uusikaupunki, Arvassalo, Finland (Hietanen 1947, 1032-1035)

Granite. Suomenniemi, Finland (Frosterus 1903,41 42; Simonen and Tyrväinen 1965)

Granite. Kuru, Niemikylä, Finland (Simonen 1952;

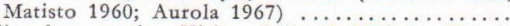
Oligoclase granite. Kisko, Orijärvi, Finland (Eskola 1914, 40-47; Rankama 1946, 29-31, 36; Eskola 1952 128-130; Tuominen 1957, 18,30; and 1961,499-515)

Granite. Vihti, Olkkola, Finland (Härme 1958 a, 45-61)

Granite. Nurmijärvi, Palojoki, Finland (Härme 1958 b)

Granite. Petäjävesi, Kintaus, Finland (Rouhunkoski 1959, $106-108)$
Granite. Petäjävesi, Kintaus, Finland Rouhunkoski Granite. Petäjävesi, Kintaus, Finland (Rouhunkosk Finland (Rouhunkoski 1959, 94-102) Granite. Petäjävesi, Pitkälä, Finland (Rouhunkoski 1959, 94-102)

Granite (»Tirilitic»). Petäjävesi, $1 \mathrm{~km}$ W from railway station, Finland (Rouhunkoski 1959, 71-90, 94-

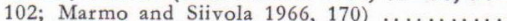

Granite ("Tirilitic»). Petäjävesi, Koskensaari, Finland (Rouhunkoski 1959, 71-90; Marmo and Siivola 1966, 170)

Granite, monzonitic. Keuruu, Finland (Marmo 1963 a;

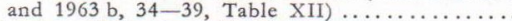

Granite. Pihtipudas, Alvajärvi, Finland (Nykänen 1962 ; and $1963,29-31)$

Granite. Hämeenkyrö, Komi, Mattila, Finland (Stigzelius 1944, 38-42; Simonen 1952, 35) .....

Granite. Hämeenkyrö, Komi, Mattila, Finland (Stigzelius 1944, 38-42; Simonen 1952, 35) .........

Granite, tourmaline-bearing. Kiihtelysvaara, Raate vaara, Pölkkylampi, Finland (Frosterus and Wilk$\operatorname{man} 1916,137-138$ )

Granite. Kaavi, Maarianvaara, Finland (Frosterus and Wilkman 1916, 128-136; Sahama and Vähätalo 1941, 67; Erämetsä, Sahama and Kanula 1943, 83, Rankama $1946,29,33,36) \ldots \ldots \ldots \ldots \ldots \ldots$ Granite. Tuusniemi, Maarianvaara, Finland (Frosterus and Wilkman 1916, 128-136; Sahama and Vähätalo 1941, 67; Erämetsä, Sahama and Kanula 1943, 83; Rankama 1946, 29, 33, 36) .............

\section{Selenium content} $\mathrm{ppb}$

\begin{tabular}{|c|c|c|}
\hline No. & Rock and locality & $\begin{array}{l}\text { Selenium } \\
\text { content } \\
\text { ppb }\end{array}$ \\
\hline 109 & $\begin{array}{l}\text { Granite, aplitic. Kalajoki, Finland (Salli } 1955 \text {; and } \\
1961,34) \ldots \ldots \ldots \ldots \ldots \ldots \ldots \ldots \ldots \ldots \ldots \ldots \ldots \ldots \ldots \ldots \ldots \ldots \ldots \ldots \ldots \ldots \\
\text { Rapakivi granites }\end{array}$ & n.d. \\
\hline 110 & $\begin{array}{l}\text { Rapakivi, syenitic. Lappeenranta, Lappee, Armila, } \\
\text { Finland (Wahl 1925,71-73; Hackman } 1934,17-24 \text {; } \\
\text { Vorma 1965) } \ldots \ldots \ldots \ldots \ldots \ldots \ldots \ldots \ldots \ldots \ldots \ldots \ldots \ldots\end{array}$ & 250 \\
\hline
\end{tabular}

111 Finland (Rankama 1946,37$)$

Viborgite. Lapinjärvi, Finland (Laitakari and Simonen 1962; and 1963, 26-31, Table III, Anal. 2) Rapakivi. Lappeenranta, Sinkkola, Finland (Wabl

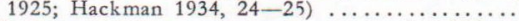

114 Biotite rapakivi, near the contact to gneiss. Mäntyharju, Ahvenisto, Finland (Savolahti 1956, 47-49) Biotite rapakivi. Suomenniemi, Finland (Pipping 1956,

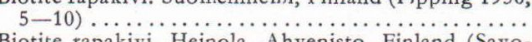

116 Biotite rapakivi. Heinola, Ahvenisto, Finland (Savolahti 1956, 47-49)

117 Biotite rapakivi, near granite porphyry. Heinola Kukkamäki, Finland (Savolahti 1956, 47-49)...

118 Pyterlite. Liljendal, Finland (Laitakari and Simonen 1962; and 1963, 26-31, Table III, Anal. 3) ....

119 Hornblende rapakivi. Suomenniemi, Finland (Pipping

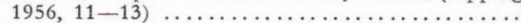

120 Viborgite. Artjärvi, Pyhäjärvi, Finland (Laitakari and

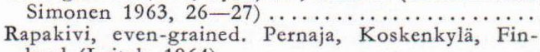

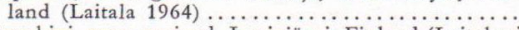

122 Rapakivi, even-grained. Lapinjärvi, Finland (Laitakari and Simonen 1962; and 1963,29-30) .........

123 Viborgite, dark. Kotka, Langinkoski, Finland (Vorma 1961, 399-400)

124 Rapakivi, even-grained. Vehmaa, Finland (Kanerva

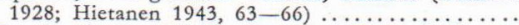

Hornblende rapakivi. Suomenniemi, Finland (Pipping

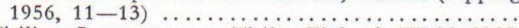

126 Tirilite. Lappeenranta, Tirilä, Finland (Wahl 1925, 69-71; Hackman 1934, 27-32) $\ldots \ldots \ldots \ldots \ldots$ Rapakivi, even-grained, small-grained. Lappeenranta, Tyysterniemi, Finland (Vorma 1965, 43, 49) Rapakivi, even-grained. Kalanti, Finland (Kanerva

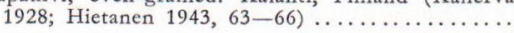

\section{Lamprophyres}

Minette. Brändö, Ava, Finland (Kaitaro 1953, 31-47) Quartz kersantite. Kuopio. Petosenmäki, Finland (Hackman 1914; Wilkman 1938, Plate I) ........ Kersantite. Parainen, Timmerkärr, Finland (Härme

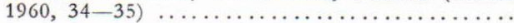

\section{Diabases}

132 Diabase. Siilinjärvi, Asikkala, Finland (Puustinen 1968,

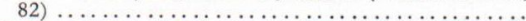

133 Diabase. Helsinki, Pitäjänmäki, Finland (Härme 1960,

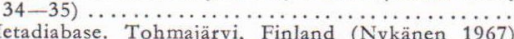

134 Metadiabase. Tohmajärvi, Finland (Nykänen 1967) 1968,82 )

136 Diabase. Salla, Jokinenä, Finland (Lauerma 1967)

137 Olivine diabase. Eurajoki, Finland (Laitakari 1925,

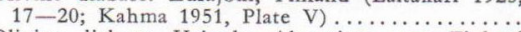

138 Olivine diabase. Heinola, Ahvenisto area. Finland (Savolahti 1956, 41-46; Laitakari 1969, 22-24)

\section{Albite diabase}

139

Albite diabase. Alatornio, Finland (Härme 1949, 1314; Mikkola 1949, 29-30; Meriläinen 1961) .... Trachyandesite (keralophyre)

140 Trachyandesite (quartz keratophyre). Ylöjärvi, from Mastojärvi to E., Kiviniemi, Finland (Simonen

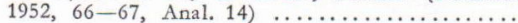

\section{Plagioclase porphyrites}

141 Plagioclase porphyrite. Pernaja, Runholm, Finland

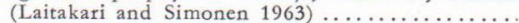

142 Plagioclase porphyrite. Ylöjärvi, Antaverkka, Fin-

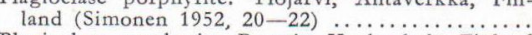

143 Plagioclase porphyrite. Pernaja, Koskenkylä, Finland (Laitakari and Simonen 1963, 13-17, Table I, No. 1)

Felspar porphyry

144 Felspar porphyry. Espoo, Finland ........... n.d.
250
180

103

80

73

60

44

36

32

30

30

30

27

23

20

19

n.d.

n.d.

n.d.

360

210

71

200
170 


\begin{tabular}{l|l|}
\hline No. & Rock and locality \\
\hline Granoplyre
\end{tabular}
Selenium content
$\mathrm{ppb}$

Granophyre, olivine-bearing, in basalt. Snxfellsnes, Laxá, Iccland (Sigurdsson 1966,77-79) ....... Pegmatites

146 Pegmatite. Mikkeli, Koskentaipale, Finland (Frosterus 1900 ; and 1903 ) .................................

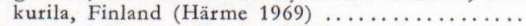

Pegmatite. Petäjävesi, $1 \mathrm{~km}$ W from railway station, Finland (Rouhunkoski 1959, 101-102)

149 Pegmatite. Porvoo, Suurpellinki, Finland (Laitala 1965)

150 Pegmatite. Petäjävesi, Kintaus, Finland (Rouhunkoski 1959, 101-102)

151 Pegmatite. Suomenniemi area, Finland (Pipping 1956)

152 Pegmatite. Peräseinäjoki, Haapaluoma, Finland ( $\mathrm{Haa}-$ pala 1966; Lappalainen and Neuvonen 1968, 61)

153 Pegmatite. Siilinjärvi, Asikkala, Finland (Puustinen $1968,16-17)$.

Pegmatite. Vimpeli, Koivujärvi, Finland (Saksela 1934) Pegmatite. Vihti, Olkkola, Finland (Härme 1960, 4547) ............................................... Pegmatite. Järvenpää, Vanhakylä, Finland (Härme

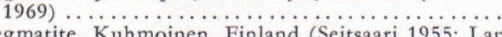
Pegmatite. Kuhmoinen, Finland (Seitsaari 1955; Lappalainen and Neuvonen 1968, 61) ............. Pegmatite. Helsinki, Malmi, Kivikko, Finland (Härme

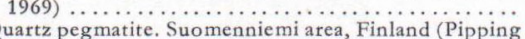
Quartz pegmatite. Suomenniemi area, Finland (Pipping Quartz pegmatite. Suomenniemi area, Finland (Pipping

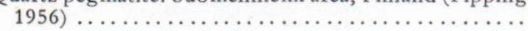

Aplitic vein rocks

162

Aplitic vein in quartzite. Kiihtelysvaara, Heinävaara, Finland (Frosterus and Wilkman 1916, 81-84)..

163 Aplitic vein. Kuopio, Neulamäki, Finland (Preston

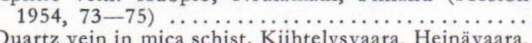
Quartz vein in mica schist. Kiihtelysvaara, Heinävaara, Finland (Frosterus and Wilkman 1916, 100-102)

165 iceous vein in kinzigite, contains cordierite. Helsinki, Käpylä, Taivaskallio, Finland (Laitala 1967) Aplitic vein. Kuopio, Jynkänlahti, Finland (Preston $1954,73-75$ )

(nelsinki, Viinik kala, Finland (Härme 1969) . ..............

Acid porphyries

168 Quartz porphyzy, glassy margin. Hamina, Huutokallio,

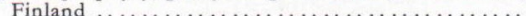

169 Quortz porphyry, middle part. Hamina, Huutokallio, Finland ............................................. land (Vorma 1965, 42-45)

171

Granite porphyry. Heinola, Ahvenisto area, Finland

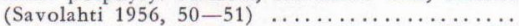
Granite porphyry. Heinola, Ahvenisto area, Finland (Savolahti 1956, 50-51) $\ldots \ldots \ldots \ldots \ldots \ldots \ldots \ldots \ldots$ Porphyry aplite. Suomennicmi area, Finland (Pipping 1956)

174 Porphyry aplite. Suomenniemi area, Finland (Pipping

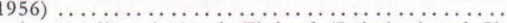
monen $1963,31,44) \ldots \ldots \ldots \ldots \ldots \ldots \ldots \ldots$ Volcanic rocks from Iceland

176 Basalt, tholeiitic, 2600 years old. Snxfellsnes, Rauda-

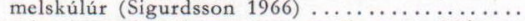
Basalt, burned red, near a small vent. Mývatn, Skútustadir (Rittman 1938; Thorarinsson $1953 \mathrm{a}$; Thorarinsson, Einarsson and Kjartansson 1959, Fig. 13) , pahochoe lava, tholeitic, year 1961. Askja (Thorarinsson and Sigvaldason 1962, 641-651; Heier, Chappell, Arriens and Morgan 1966, 427-

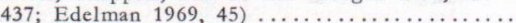
Basalt, altered, contains pyrite, Pleistocene. Snzfellsnes, Lýsuskard (Sigurdsson 1966) .................... Basaltic pillow lava, porpbyritic. Askja, Oskjuvatn (Thorarinsson and Sigvaldason 1962, 641, Heier,

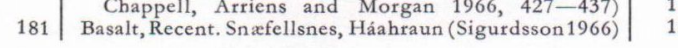

n.d.

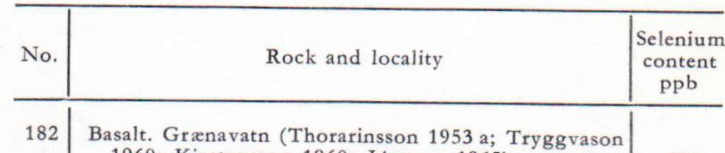

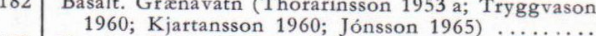
Basalt, olivine-bearing, 2600 years old. Snxfellsnes,

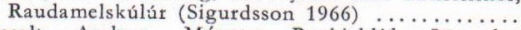
184 Basalt, Aa-lava. Mývatn, Reykjahlid, Jörundur

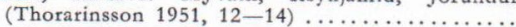
185 Basalt, altered partly to clay minerals. Akyreyri, Valagil (Thorarinsson, Einarsson and Kjartansson 1959) ...........

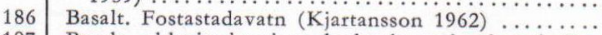

187 Basalt, chlorite-bearing, hydrothermaly altered. Setberg, Grund (Sigurdsson 1966)

188 Basalt, dense. Granavatn (Kjartansson 1960; Jónsson

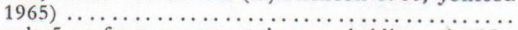

189 Basalt, $5 \mathrm{~cm}$ from contact against an obsidian vein (No. 207), Tertiary. Varmaland, Hraunfossar (Thorarinsson, Einarsson and Kjartansson 1959)

190 Basalt, olivine-beating, columnar flow, $2 \mathrm{~m}$ thick. Snafellsnes, Gríshólsa (Sigurdsson 1966)

191 Ankaramite. Skógar, Hvammsmúli, Pöst (Kjartansson 1960; Steinhorsson 1964) .....

192 Basalt, pillow lava (under No. 190). Snæfell . . .

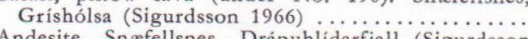
Andesite. Snæfellsnes, Drápuhlidarfjall (Sigurdsson

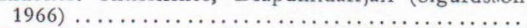

194 Hyperstehne andesite. Mývatn, Hverfjall (Thorarinsson, Einarsson and Kjartansson 1959, Fig. 13

195 Andesite ("Icelandite»). Snefellsnes, Háahraun (Si-

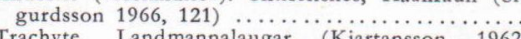

196 Trachyte. Landmannalaugar (Kjartansson 1962;

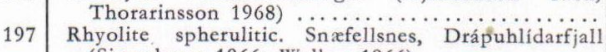
(Sigurdsson 1966; Walker 1966) ................. 1966; Walker 1966) Drápublídarfjall (Sigurdsson
(96)

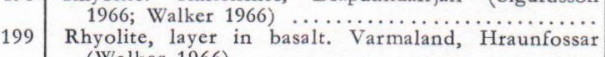

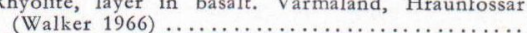

200 Rhyolite. Snæfellsnes, Lýsuskard (Sigurdsson 1966)

201 Pumice. Near Askja, Viti eruption year 1875 (Thorarinsson and Sigvaldason 1962, 642; Heier, Chappell, Arriens and Morgan 1966, 427-437;

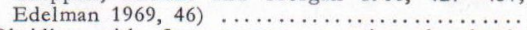

202 Obsidian with flow texture, contains abundantly vesicles. Varmaland, Hraunfossar (Walker 1966) 203 Obsidian Mývatn, Skútustadir (Thorarinsson and Kjartansson 1959, Fig. 13; Walker 1966)

204 Obsidian. Frostastadavatn (Kjartansson 1962; Walker

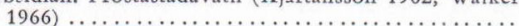

205 Obsidian. Snæfellsnes, Drápuhlidarfjall (Sigurdsson

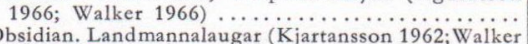
Obsidian. Landmannalaugar (Kjartansson 1962; Walker

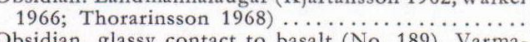

207 Obsidian, glassy contact to basalt (No. 189). Varmaand, Hraunfossar (Walker 1966)

208 Ignimbrite. Thórsmörk (Thorarinsson $1969,139-154$ )

209 Ignimbrite. Thórsmörk (Thorarinsson 1969, 139-154)

210 Agglomerate, rhyolitic. Snafellsnes, Setberg, Hrafnagil (Sigurdsson 1966)

211 Basaltic ash, 10000 years old. Akyreyri (Thorarinsson, Einarsson and Kjartansson 1959, Fig. 10)

212 Basaltic ash, 500 years old. Akyreyri (Thorarinsson, Einarsson and Kjartansson 1959, Fig. 10) .......

213 Rhyolitic ash, Hekla III. Akyreyri (Thorarinsson $1953 \mathrm{~b}$; Thorarinsson, Einarsson and Kjartansson

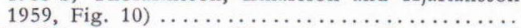

214 Rhyolitic ash. Akyreyri (Thorarinsson, Einarsson and Kjartansson 1959, Fig. 10) ..............................
Rhyolitic ash. Askja (Thorarinsson and Sigvaldason

216 Rhyolitic ash. Thórsmörk (Thorarinsson 1969, 139-

217 Tuff, chloritized. Landmannalaugar (Kjartansson 1962;

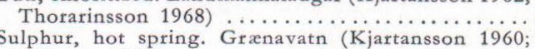

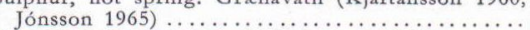
Geyserite. Great Geysir (Kjartansson 1960) ................ Opal. Sn xfellsnes, Setberg (Sigurdsson 1966) .......
Travertine. Snxfellsnes, Lýsuskard (Sigurdsson 1966)

\section{Miscellaneous}

lainen 1968) .......

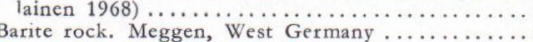

n.d. 


\section{REFERENCES}

Aurola, E. (1967) Kurun alueen rakennuskivet. Geotek. julk. 71.

Behrend, F. and Berg, G. (1927) Chemische Geologie. Verlag von F. Enke, Stuttgart.

Brunefelt, A. O. and Steinnes, E. (1967) Determination of selenium in standard rocks by neutron activation analysis. Geochim. Cosmochim. Acta 31, 283-285.

Clarke, F. W. and Washington, H. S. (1924) The composition of the earth's crust. U. S. Geol. Surv., Prof. Pap. 127, 27, 83, 89-93.

Davidson, D. F. and Powers, H. A. (1959) Selenium content of some volcanic rocks from western United States and Hawaiian Islands. U. S. Geol. Surv., Bull. $1084-\mathrm{C}, 69-81$.

Edelman, N. (1969) Island, vulkanernas ö. Nordenskiöld-samfundets tidskrift 29, $40-49$.

Erämetsä, O., Sahama, Th. G. and Kanula, V. (1943) Spektrographische Bestimmungen an Rubidium und Caesium in einigen finnischen Mineralen und $\mathrm{Ge}$ steinen. Bull. Comm. géol. Finl. 128, 80-86.

Eskola, P. (1914) On the petrology of the Orijärvi region in southwestern Finland. Fennia 37: 3, 1-277.

- (1952) A discussion of domes and granites and ores. Bull. Comm. géol. Finl. 157, 125-144.

Frosterus, B. (1900) General geological map of Finland, 1: 400000 , Sheet C2, Mikkeli. Geologinen tutkimuslaitos, Otaniemi.

- (1903) Vuorilajikartan selitys C2, Mikkeli. General geological map of Finland, 1: 400000 . Geologinen tutkimuslaitos, Otaniemi.

- and Wilkman, W. W. (1916) Beskrifning till bergartskartan D3, Joensuu. Résumé en francais. General geological map of Finland, 1: 400000 . Geologinen tutkimuslaitos, Otaniemi.

Goldschmidt, V. M. (1958) Geochemistry. Clarendon Press, Oxford.

- and Hefter, O. (1933) Zur Geochemie des Selens. Nachr. Ges. Wiss. Göttingen, Math.-Phys. Kl. III, $35,245-252$.

- and Strock, L. W. (1935) Zur Geochemie des Selens II. Nachr. Ges. Wiss. Göttingen, Math.-Phys. K1. IV, NF. 1, 123-142.

HaApala, I. (1966) On the granatic pegmatites in the Peräseinäjoki-Alavus area, South Pohjanmaa, Finland. Bull. Comm. géol. Finl. 224.

HACKMAN, V. (1905) Die chemische Beschaffenheit von Eruptivgesteinen Finlands und der Halbinsel Kola im Lichte des neuen amerikanischen Systemes. Bull. Comm. géol. Finl. 15.

- (1914) Über Camptonitgänge im mittleren Finnland. Bull. Comm. géol. Finl. 42.
HaCKMAN, V. (1934) Das Rapakiviwirandgebiet der Gegend von Lappeenranta (Willmanstrand). Bull. Comm. géol. Finl. 106.

HALL, W. (1936) Kiviteollisuus. Otava, Helsinki.

Heier, K. S., Chappell, B. W., Arriens, P. A. and Morgan, J. W. (1966) The geochemistry of four Icelandic basalts. Nor. Geol. Tidsskr. 46, 427-437.

Hietanen, A. (1943) Über des Grundgebirge des Kalanti gebietes im südwestlichen Finnland. Ann. Acad. Sci. Fenn., Ser. A, III, 6.

- (1947) Archean geology of the Turku district in southwestern Finland. Bull. Geol. Soc. Am. 58, 1019-1084.

Hunma, A., Salli, I. and Matisto, A. (1952 a) Geological map of Finland, 1: 100000 , Sheet 2122, Ikaalinen. Geologinen tutkimuslaitos, Otaniemi.

- (1952 b) Kallioperäkartan selitys 2122, Ikaalinen. English summary: Explanation to the map of rocks. Geological map of Finland, 1: 100000 . Geologinen tutkimuslaitos, Otaniemi.

Härme, M. (1949) On the stratigraphical and structural geology of the Kemi area, northern Finland. Bull. Comm. géol. Finl. 147.

- (1953) Geological map of Finland, 1: 100 000, Sheet 2042, Karkkila. Geologinen tutkimuslaitos, Otaniemi.

- (1954) Structure and stratigraphy of the Mustio area, southern Finland. Bull. Comm. géol. Finl. 166, 29-48.

- $(1958 \mathrm{a})$ Examples of the granitization of plutonic rocks. Bull. Comm. géol. Finl. 180, 45-64.

- (1958 b) General geological map of Finland, 1: 400000 , Sheet B1, Turku. Geologinen tutkimuslaitos, Otaniemi.

- (1960) Kivilajikartan selitys B1, Turku. English summary. General geological map of Finland, 1: 400000. Geologinen tutkimuslaitos, Otaniemi.

- (1965) On the potassium migmatites of southern Finland. Bull. Comm. géol. Finl. 219.

- (1969) Geological map of Finland, 1: 100 000, Sheet 2043, Kerava. Geologinen tutkimuslaitos, Otaniemi.

Jónsson, J. (1965) Bergsprungur og misgengi í nagrenni Reykjavíkur. Náttúru Frædingurinn 35, 75-95.

Kaнma, A. (1951) On contact phenomena of the Satakunta diabase. Bull. Comm. géol. Finl. 152.

Kaitaro, S. (1953) Geologic structure of the late preCambrian instrudives in the Ava area, Aland Islands. Bull. Comm. géol. Finl. 162.

- (1956) Geological map of Finland, 1: 100 000, Sheet 2044, Riihimäki. Geologinen tutkimuslaitos, Otaniemi.

Kanerva, I. (1928) Über das Rapakiwigebiet von Vehmaa im südwestlichen Finnland. Fennia 50: 40.

KJartansson, G. (1960) Geological map of Iceland, 1: 250000 , Sheet 3, Sudvesturland. Museum of Natural History, Reykjavik. 
KJartansson, G. (1962) Geological map of Iceland, 1: 250000 , Sheet 6, Midsuđurland. Museum of Natural History, Reykjavik.

KoljONEN, T. (1965) Seleenin esiintymisestä suomalaisissa kivissä. Unpubl. manuscr., Arch. Dep. Geol. Miner., Univ. Helsinki.

Kurkr, J. (1964) Niemilahden malmilohkareen ja Kurolahden-Rauhamäen alueen ultraemäksisten pahkujen petrografiaa. Unpubl. manuscr., Arch. Dep. Geol. Miner., Univ. Helsinki.

LAITAKARI, A. (1925) Uber das jotnische Gebiet von Satakunta. Bull. Comm. géol. Finl. 73.

LAITAKARI, I. (1969) On the set of olivine diabase dikes in Häme, Finland. Bull. Comm. géol. Finl. 241.

- and Simonen, A. (1962) Geological map of Finland, 1: 100 000, Sheet 3022, Lapinjärvi. Geologinen tutkimuslaitos, Otaniemi.

- (1963) Kallioperäkartan selitys 3022, Lapinjärvi. English summary: Explanation to the map of rocks. Geological map of Finland, 1: 100000 . Geologinen tutkimuslaitos, Otaniemi.

Laitala, M.(1964) Geological map of Finland, 1: 100 000, Sheet 3021, Porvoo. Geologinen tutkimuslaitos, Otaniemi.

- (1965) Geological map of Finland, 1: 100 000, Sheet 3012, Pellinki. Geologinen tutkimuslaitos, Otaniemi.

- (1967) Geological map of Finland, 1: 100 000, Sheet 2034, Helsinki. Geologinen tutkimuslaitos, Otaniemi.

Lappalainen, R. and Neuvonen, K. J. (1968) Trace elements in some Finnish pegmatitic potassium feldspars. Bull. Geol. Soc. Finl. 40, 59-64.

Lauerma, R. (1967) Geological map of Finland, 1: 100000 , Sheets 4621 - 4623, Salla. Geologinen tutkimuslaitos, Otaniemi.

LenIJÄrvi, M. (1960) The alkaline district of Iivaara, Kuusamo, Finland. Bull. Comm. géol. Finl. 185.

Luttrel, S. V. (1959) Annotated bibliography of the geology of selenium. U. S. Geol. Surv., Bull. 1019-M, 867-972.

Marmo, V. (1963 a) Geological map of Finland, 1: 100 000, Sheet 2232, Keuruu. Geologinen tutkimuslaitos, Otaniemi.

- (1963 b) Kallioperäkartan selitys 2232, Keuruu. English summary: Explanation to the map of rocks. Geological map of Finland, 1: 100000 . Geologinen tutkimuslaitos, Otaniemi.

- (1965 a) Geological map of Finland, 1: 100 000, Sheet 2214, Virrat. Geologinen tutkimuslaitos, Otaniemi.

- (1965 b) Kallioperäkartan selitys 2214, Virrat. English summary: Explanation to the map of rocks. Geological map of Finland, 1: 100 000. Geologinen tutkimuslaitos, Otaniemi.

- and SirvolA, J. (1966) On the bariumcontent of some granites of Finland. C. R. Soc. Géol. Finl. 38, 169-172.
Matisto, A.(1960) Geological map of Finland, 1: 100 000, Sheet 2213, Kuru. Geologinen tutkimuslaitos, Otaniemi.

- (1961) Geological map of Finland, 1: 100 000, Sheet 2123, Tampere. Geologinen tutkimuslaitos, Otaniemi.

- (1962) Ortoklaasipitoisista porfyyrisistä granodioriiteista - On some orthoclase-bearing porphyritic granodiorites. Geologi 14, 118-121.

- (1968) Die Meta-Arkose von Mauri bei Tampere. Bull. Comm. géol. Finl. 235.

Merilärnen, K. (1961) Albite diabases and albitites in Enontekiö and Kittilä, Finland. Bull. Comm. géol. Finl. 195.

Miknola, A. (1949) On the geology of the area North of the Gulf of Bothnia. Bull. Comm. géol. Finl. 146.

Noddack, I. and Noddack, W. (1936) Die Verteilung der nutzbaren Metalle in der Erdrinde. Angew. Chem. $49,1-5$.

Nuutilainen, J. (1968) On the geology of the Misi iron ore province, northern Finland. Ann. Acad. Sci. Fenn., Ser. A, III, 96.

NyKänen, O. (1962) Geological map of Finland, 1: 100 000, Sheet 2334, Kinnula. Geologinen tutkimuslaitos, Otaniemi.

- (1963) Kallioperän selitys 2334, Kinnula. English summary: Explanation to the map of rocks. Geological map of Finland, 1: 100000 . Geologinen tutkimuslaitos, Otaniemi.

- (1967) Geological map of Finland, 1: 100 000, Sheets 4232 -4234, Tohmajärvi. Geologinen tutkimuslaitos, Otaniemi.

NÄYккI, O. (1964) Kemin-Kivaloiden emäksinen kompleksi. Vihreäkivet ja kerroksellinen magmakivisarja. Unpubl. thesis, Arch. Dep. Geol. Miner., Univ. Helsinki.

Pipping, F. (1956) Rapakivimassivet i Suomenniemi. Unpubl. manuscr., Arch. Dep. Geol. Miner., Univ. Helsinki.

Preston, J. (1954) The geology of the Pre-Cambrian rocks, of the Kuopio district. Ann. Acad. Sci. Fenn., Ser. A, III, 40.

PuUstinen, K. (1968) Siilinjärven apatiittimuodostuman geologiasta. Unpubl. thesis, Arch. Dep. Geol. Miner., Univ. Helsinki.

- (1969) The carbonatite of Siilinjärvi in the Precambrian of eastern Finland. Lithos 3, 89-92.

- (1971) Geology of the Siilinjärvi carbonatite complex, eastern Finland. Bull. Comm. géol. Finl. 249.

Rankama, K. (1943) The niobium and tantalum content of three Finnish Archean granites (A preliminary report). Bull. Comm. géol. Finl. 128, 34-39.

- (1944) On the geochemistry of tantalum. Bull. Comm. géol. Finl. 133. 
RANKAMA, K. (1946) On the geochemical differentiation in the earth's crust. Bull. Comm. géol. Finl. 137.

Rittmann, A. (1938) Die Vulkane am Myvatn. Bull. Volcanol. II, 4, 1-38.

Roununkoskr, P. (1959) Petäjäveden alueen geologiaa. Unpubl. thesis, Arch. Dep. Geol. Miner., Univ. Helsinki.

Sahama, Th. G. and Vähätalo, V. (1941) X-ray spectrographic study of the rare earths in some Finnish eruptive rocks and minerals. Bull. Comm. géol. Finl. 126, 50-83.

SAKsela, M. (1934) General geological map of Finland, 1: 400000 , Sheet B3, Vaasa. Geologinen tutkimuslaitos, Otaniemi.

SALLI, I. (1955) Geological map of Finland, 1: 100000 , Sheets 2413-2431, Kalajoki-Ylivieska. Geologinen tutkimuslaitos, Otaniemi.

- (1961) Kallioperäkartan selitys 2413-2431-2433, Kalajoki-Ylivieska-Haapavesi. English summary: Explanation to the map of rocks. Geological map of Finland, 1: 100 000. Geologinen tutkimuslaitos, Otaniemi.

- (1963) Geological map of Finland, 1: 100 000, Sheet 2343, Reisjärvi. Geologinen tutkimuslaitos, Otaniemi.

- (1965) Kallioperäkartan selitys 2432-2434, Pyhäjoki -Vihanti. English summary: Explanation to the map of rocks. Geological map of Finland, 1: 100 000. Geologinen tutkimuslaitos, Otaniemi.

Savolahtr, A. (1956) The Ahvenisto massif in Finland. The age of the surrounding gabbro-anorthosite complex and the crystallization of rapakivi. Bull. Comm. géol. Finl. 174.

- (1966) The differentiation of gabbro-anorthosite intrusions and the formation of anorthosites. Bull. Comm. géol. Finl. 222, 173-197.

SeitsaAri, J. (1951) The schist belt Northeast of Tampere in Finland. Bull. Comm. géol. Finl. 153.

- (1955) Geological map of Finland, 1: 100 000, Sheet 2012, Perniö. Geologinen tutkimuslaitos, Otaniemi.

Sigurdsson, H. (1966) Geology of the Setberg area, Snxfellsnes, western Iceland. Greinar IV: 2, Vísindafélag Íslendinga, Reykjavik.

Srmonen, A. (1941) Orbicular rocks in Kemijärvi and Esbo. Bull. Comm. géol. Finl. 126, 107-140.

- (1952) Kallioperäkartan selitys 2124, ViljakkalaTeisko. English summary: Explanation to the map of rocks. Geological map of Finland, 1: 100 000. Geologinen tutkimuslaitos, Otaniemi.

- (1966) Orbicular rock in Kuru, Finland. Bull. Comm. géol. Finl. 222, 93-107.

- and Trrväinen, A. (1965) Geological map of Finland, 1: 100 000, Sheet 3132, Savitaipale. Geologinen tutkimuslaitos, Otaniemi.
Sindeeva, N. D. (1964) Mineralogy and types of deposits of selenium and tellurium. Interscience Publishers, New York-London-Sydney.

Sternthorsson, S. (1964) The ankaramites of Hvammsmuli, Eyjafjöll, southern Iceland. Acta Nat. Isl. II: 4.

Stigzelius, H. (1944) Über die Erzgeologie des Viljakkala-Gebietes im südwestlichen Finnland. Bull. Comm. géol. Finl. 134.

Thorarinsson, S. (1951) Laxárgljúfur and Laxárhraun. A tephrochronological study. Geogr. Ann. Stockh. 33, $1-88$.

- (1953 a) Grænavatn and Gestssteđavatn. Geogr. Tidsskr. 52, 292-301.

- (1953 b) The crater groups of Iceland. Bull. Volcanol. 14, $1-44$.

- (1968) Skaftáreldar og Lakagigar. Náttrúufræđingurinn 37: $1-2,27-57$.

-(1969) A Pleistocene ignimbrite in Thórsmórk. Náttúrufræđingurinn 39: 3-4, 139-155.

- Einarsson, T. and KJartansson, G. (1959) On the geology and geomorphology of Iceland. Geogr. Ann. Stockh. 41, 135-169.

- and Sigvaldason, G. E. (1962) The eruption in Askja, 1961. A preliminary report. Am. J. Sci. 160, $641-651$.

Tischendorf, G. (1966) Zur Verteilung des Selens in Sulfiden. Freib. Forschungsh. C 208.

Tryggvason, T. (1960) The gabbro bombs at Lake Grænavatn. Bull. Geol. Inst. Upps. 38, 1-5.

Tuominen, H. V. (1957) The structure of an Archean area: Orijärvi, Finland. Bull. Comm. géol. Finl. 177.

- (1961) The structural position of the Orijärvi granodiorite and the problem of synkinematic granites. Bull. Comm. géol. Finl. 196, 499-515.

Turektan, K. K. and Wedepohl, K. H. (1961) Distribution of the elements in some major units of the Earth's crust. Geol. Soc. Am. Bull. 72, 175-192.

Vernadsky, W. J. (1924) La Géochimie. Libraire Félix Alcan, Paris.

Vinogradov, A. P. (1962) Average contents of chemical elements in the principal types of igneous rocks of the earth's crust. Geochem. 7, 641-664.

Vogr, J. H. L. (1898) Ueber die relative Verbreitung der Elemente, besonders der Schwermetalle, und über die Concentration des ursprünglich fein vertheilten Metallgehaltes zu Erzlagerstätten. Z. prakt. Geol., $225-238$.

Vorma, A. (1961) A new apophyllite occurrence in the Viipuri rapakivi area. Bull. Comm. géol. Finl. 196, $399-404$.

- (1964) Geological map of Finland, 1: 100 000, Sheet 3134, Lappeenranta. Geologinen tutkimuslaitos, Otaniemi. 
Vorma, A. (1965) Kallioperäkartan selitys 3134, Lappeenranta. English summary: Explanation to the map of rocks. Geological map of Finland, 1: 100000 . Geologinen tutkimuslaitos, Otaniemi.

VÄISÄNEN, S. (1967) Itipiluan ultraemäksinen massiivi ja siihen liittyvä kromiitti. Unpubl. manuscr., Arch. Dep. Geol. Miner., Univ. Helsinki.

WaHL, W. (1925) Die Gesteine des Wiborger Rapakiwigebietes. Fennia 45: 20.

WALker, G. P. L. (1966) Acid volcanic rocks in Iceland. Bull. Volcanol. 29, 375-406.
WedePoHL, K. H. (1969) Handbook of geochemistry I. Springer-Verlag, Berlin-Heidelber-New York.

Wilkman, W. W. (1931) Kivilajikartan selitys C4, Kajaani. General geological map of Finland, 1: 400000. Geologinen tutkimuslaitos, Otaniemi.

- (1938) Kivilajikartan selitys C3, Kuopio. English summary. General geological map of Finland, 1: 400 000. Geologinen tutkimuslaitos, Otaniemi.

Manuscript received, February 29, 1972. 